\title{
Canes for knee osteoarthritis: is a randomised trial necessary?
}

\author{
Kåre B Hagen
}

In this issue of the Annals, Jones et al (pp 172) report the results of a randomised clinical trial (RCT) of canes for knee osteoarthritis. Current recommendations on the management of hip and knee osteoarthritis emphasise non-pharmacological interventions, with sticks or canes universally recommended in existing guidelines. ${ }^{2}$ According to the Osteoarthritis Research Society International (OARSI) recommendations for the management of hip and knee osteoarthritis one of 25 treatment propositions recommended is 'Walking aids can reduce pain in patients with hip and knee OA. Patients should be given instruction in the optimal use of a cane or crutch in the contralateral hand. Frames or wheeled walkers are often preferable for those with bilateral disease. ${ }^{3}$ In the National Institute for Health and Clinical Excellence (NICE) guideline for care and management of osteoarthritis in adults, assistive devices (such as walking sticks) are considered as adjunct treatments. ${ }^{4}$ While both the OARSI and the NICE recommendations acknowledge the paucity of well-designed clinical trials in this area, there appears to be a high degree of expert consensus that walking aids can reduce pain in patients with hip and knee osteoarthritis. One might then ponder the need for a randomised trial on canes for knee osteoarthritis.

RCT provide the most unbiased evidence about the benefits and harms of medical interventions. However, sometimes when there are clear indications from observational studies that an intervention has a dramatic beneficial effect, a RCT is considered unnecessary and unethical. For example, although the evidence for the effectiveness of joint replacement surgery for severe knee and hip osteoarthritis is based substantially on uncontrolled observational studies and cohort studies in which outcomes

National Resource Center for Rehabilitation,

Diakonhjemmet Hospital, Oslo, Norway

Correspondence to Kåre B Hagen, National Resource Center for Rehabilitation in Rheumatology, Diakonhjemmet Hospital, PO box 23,

Vinderen, Oslo, Norway;

k.b.hagen@medisin.uio.no have been compared with standard medical care, most people will agree that a RCT comparing surgery with the standard medical care of the present is unethical and unnecessary. Although the use of canes and sticks goes back as far as ancient Egypt, there was until now no clinical research to support or refute its effectiveness. The only empirical evidence comes from a biomechanical study of knee moments of force following the use of a cane in the contralateral hand. ${ }^{5}$ In a fluctuating condition such as knee osteoarthritis, individual cases and clinical experience of an intervention with at best a moderate effect are liable to be misleading, and a RCT on canes for knee osteoarthritis is therefore needed.

In the RCT by Jones et al, ${ }^{1} 64$ patients attending an outpatient rheumatology clinic in Sao Paulo, Brazil, were enrolled. Inclusion criteria were the diagnosis of knee osteoarthritis according to the American College of Rheumatology criteria, knee pain score between 3 and 7 (on a $0-10$ visual analogue scale), stable doses of non-steroidal anti-inflammatory drugs, and no regular physical exercise or use of canes in the months before the study. Each participant in the intervention group received a wooden cane with a $\mathrm{T}$-shaped handle and instruction in how to use it at the start of the intervention and after 1 month, and took the cane home for dayto-day use for 2 months. The participants in the control group were instructed not use any gait auxiliary device for 2 months, but otherwise maintain their normal lives including treatment as usual. The primary endpoint was pain on a $0-10$ visual analogue scale. Mean differences in pain between the two groups were 0.77 after 1 month and 2.11 after 2 months, both in favour of the group who used canes, corresponding to a $\omega$-squared effect size of 0.18. As I am not familiar with the $\omega$-squared effect size, I have calculated the more frequently used Cohens's effect size for the mean difference at 2 months follow-up (mean difference/pooled SD at baseline). Assuming that the pooled SD at baseline was 1.1, the Cohen's effect size for pain is 0.9 , which is higher than all other non-surgical treatment modalities offered to patients with knee osteoarthritis. $^{2}$ Interestingly, also some important secondary outcomes, among them the Lequesne index and consumption of nonsteroidal inti-inflammatory drugs, were significantly different in favour of the intervention group. However, we should keep in mind that these are the results from the first published RCT on canes, and that the magnitude of effect needs to be confirmed in further trials.

While waiting for the results from more trials on canes, we can look to Hill's proposed list of factors strengthening confidence in inferences, ${ }^{6}$ ie, temporal relation (treatment precedes effect), strength of relation (eg, magnitude of effect), plausibility (based on current understanding of disease mechanism), consistency (across settings and methods), coherence (with knowledge of related treatments), doseresponse relation and specificity (treatment causes the effect). Even though these criteria were designed for interpreting observational studies they may still be useful in this context. First, there is definitely a temporal relationship, ie, is the treatment preceded the effect. Second, considering the magnitude of the treatment effect on pain mentioned above, the effect size is considerable. Third, as this is the first trial on canes, there is no empirical evidence for the consistency of results across settings and methods. Fourth, the findings of the trial are coherent with knowledge of related treatments, as there are some trials showing a favourable outcome of assistive devices for osteoarthritis in the hand and considering the fact that canes and walking frames are recommended in most clinical guidelines. Regarding specificity, it is not possible to determine whether the observed effect was caused by the treatment in itself, and not the procedure of the treatment, ie, the magnitude of the placebo effect is unknown. Neither do we know anything about any dose-response relation.

Hill's seventh and last criterion, which concerns whether the results are plausible based on the current understanding of disease mechanism, is interesting and might be worth discussing. Current research findings show an association between an increased body mass index and a greater risk of both incident knee osteoarthritis and knee osteoarthritis progression, while the association between an increased body mass index and the risk of either incident or progressive hip osteoarthritis is weak or non-existent. ${ }^{7}$ Knee malalignment seems to increase the risk of osteoarthritis disease progression, most likely by directing the 
body weight forces to a specific tibiofemoral compartment. This might explain why the knee joint is particularly vulnerable to mechanical stress. There is also some evidence suggesting that the symptoms from an osteoarthritis joint can be improved if the mechanical stress is normalised by surgical procedures such as osteotomy or distraction. ${ }^{8}$ Therefore, the reduction or change in mechanical loads in the osteoarthritis knee with a cane during walking might explain the symptom improvement found in the trial by Jones et al. ${ }^{1}$

It is interesting that while the use of canes and sticks goes back to ancient Egypt, the first randomised trial on the effects of canes for knee osteoarthritis was not published until now. An editorial published in the Annals of the Rheumatic Diseases 11 years ago raised the question: 'do patients with osteoarthritis get the clinical research they need?'. 9 The question was posed in response to a study showing that pharmacological (59.1\%) and surgical $(25.6 \%)$ studies represented the majority of the literature. ${ }^{10}$ Later the same researchers reported that research into surgery and educational interventions were the highest priorities for patients. ${ }^{11}$ These findings led the investigators to conclude that there is a mismatch between the majority of published research on interventions for knee osteoarthritis and the degree of interest in these interventions among patients. ${ }^{12}$ Although we do not know if patients have the same priorities now, we do know that there is an increasing amount of evidence supporting non-pharmacological interventions for knee osteoarthritis. However, there are still many important unanswered research questions related to non-pharmacological approaches. For example, there is limited evidence for the optimal content, dosage and mode of delivery of exercises for knee osteoarthritis.

While most international guidelines for knee osteoarthritis consider non-pharmacological and pharmacological treatments as equally important, ${ }^{2}$ the NICE guideline defines three non-pharmacological interventions as core approaches. ${ }^{4}$ The core approaches are:

- Access to appropriate oral and written information, to enhance the patient's understanding of their condition and to counter misconceptions.

- Activity and exercise, including local muscle strengthening and general aerobic fitness.

- Interventions to achieve weight loss if the patient is overweight or obese.

Pharmacological and other non-pharmacological interventions are considered as adjunct to the core approaches in the NICE guideline. Although we know little about how people with osteoarthritis are treated, there is some evidence suggesting that non-pharmacological management strategies are underused by physicians in both primary and secondary care. ${ }^{13} 14 \mathrm{We}$ therefore also need to move the research agenda from the stage of 'intention' to the stage of 'implementation'. There is a need for more research on effective strategies to help people with painful knee osteoarthritis to start to exercise and to lose weight. There is no magic bullet for behavioural change. Exercise and weight loss appear to be effective for reducing pain for overweight people with osteoarthritis, but both are very difficult and probably require multiple strategies to be effective. Most patients find it difficult to exercise when joints are already very painful, and it is difficult to lose weight if you cannot exercise. Resolution of these issues will require close collaboration between physicians from different specialities and non-physician health professionals, both in clinical care and in research.

Competing interests None.

Provenance and peer review Commissioned; externally peer reviewed.

Accepted 21 October 2011

Ann Rheum Dis 2012;11:159-160.

doi:10.1136/ard.2011.200367

\section{REFERENCES}

1. Jones A, Silva PG, Rodrigues Silva AM, et al. Impact of cane use on pain, function, general health and energy expenditure during gait in patients with knee osteoarthritis: a randomized controlled trial. Ann Rheum Dis 2011:71:172-79.

2. Zhang W, Moskowitz RW, Nuki G, et al. OARSI recommendations for the management of hip and knee osteoarthritis, part l: critical appraisal of existing treatment guidelines and systematic review of current research evidence. Osteoarthritis Cartilage 2007; 15:981-1000

3. Zhang W, Moskowitz RW, Nuki G, et al. OARSI recommendations for the management of hip and knee osteoarthritis, part II: OARSI evidence-based, expert consensus guidelines. Osteoarthritis Cartilage 2008;16:137-62.

4. National Institute for Health and Clinical Excellence. Osteoarthritis: national clinical guideline for care and management in adults. London: NICE, 2008. www. nice.org.uk/CG059.

5. Chan GN, Smith AW, Kirtley C, et al. Changes in knee moments with contralateral versus ipsilateral cane usage in females with knee osteoarthritis. Clin Biomech (Bristol, Avon) 2005;20:396-404.

6. Hill $\mathbf{A B}$. The environment and disease: association or causation? Proc $R$ Soc Med 1965;58:295-300

7. Reijman M, Pols HA, Bergink AP, et al. Body mass index associated with onset and progression of osteoarthritis of the knee but not of the hip:the Rotterdam Study. Ann Rheum Dis 2007;66:158-62.

8. Intema F, Van Roermund PM, Marijnissen AC, et al. Tissue structure modification in knee osteoarthritis by use of joint distraction: an open 1-year pilot study. Ann Rheum Dis 2011;70:1441-6.

9. Gøtzsche PC. Do patients with osteoarthritis get the clinical research they need? Ann Rheum Dis 2000;59: 407-8.

10. Chard JA, Tallon D, Dieppe PA. Epidemiology of research into interventions for the treatment of osteoarthritis of the knee joint. Ann Rheum Dis 2000:59:414-18

11. Tallon D, Chard J, Dieppe P. Exploring the priorities of patients with osteoarthritis of the knee. Arthritis Care Res 2000;13:312-19.

12. Tallon D, Chard J, Dieppe P. Relation between agendas of the research community and the research consumer. Lancet 2000;355:2037-40.

13. Scarpa R, Sarzi-Puttini P, Cimmino MA, et al. Analysis of pharmacologic and nonpharmacologic prescription patterns of general practitioners and specialists in the AMICA study. Semin Arthritis Rheum 2005;35(1 Suppl 1):24-30.

14. Mitchell HL, Carr AJ, Scott DL. The management of knee pain in primary care: factors associated with consulting the GP and referrals to secondary care. Rheumatology (Oxford) 2006;45:771-6. 